\title{
Estrogens and Gastroparesis: A Clinical Relevance
}

\author{
Jaladanki N. Rao
}

Published online: 27 April 2013

(C) Springer Science+Business Media New York 2013

Normal gastric emptying requires coordinated function of upper stomach fundus, lower body, antrum, and pylorus. Gastric motility is controlled by a very complex set of neuornal and hormonal signals. Several cell types, including the extrinsic innervation to the stomach, enteric nerves, glia, smooth muscle cells, interstitial cell of Cajal (ICC), and immune cells are known to be responsible for normal gastric motility functions. It has been shown that, in diabetic as well as idiopathic patients, the lack of functional coordination between these cell types may have been lost; therefore, the motility of the stomach is impaired and thus leads to abnormal gastric emptying [1].

Gastroparesis is a chronic motility disorder defined as delayed gastric emptying of solids and liquids in the absence of obstruction. The most common forms include idiopathic, diabetic, and post-surgical complications [1-3]. The majority of idiopathic patients are either obese or overweight [3]. A significant limitation to developing targeted therapy for gastroparesis is a lack of understanding of the pathological and cellular etiology. Women, in particular the younger population with a mean age of 33-44 years, are more susceptible for gastroparesis than age-matched men [1-3]. Ovarian hormonal influence has been proposed as a major contributor to changes observed in gastric emptying in both health and disease states [4]. Most of our understanding for gastroparesis has come from animal studies focused on diabetic, hyperlipidemia, and

\section{J. N. Rao}

Department of Surgery, University of Maryland School of Medicine, Baltimore, MD 21201, USA

\section{J. N. Rao $(\bowtie)$}

Department of Surgery, Baltimore Veterans Affairs Medical Center, 10 North Greene Street, Baltimore, MD 21201, USA e-mail: jrao@umaryland.edu oxidative stress-induced gastroparesis (reviewed in detail in [4]). Most recently, Ravella et al. [5] demonstrated that lack of sex hormones can lead to impaired nitrergic function leading to delayed gastric emptying. Studies published in experimental diabetic rats have demonstrated that serum estrogens are elevated, and this may be somewhat harmful for altered gastric emptying [6]. In contrast, Mankhey et al. [7] demonstrated that diabetes induction reduced serum estrogen levels and that supplementation of this hormone improved renal function. It is important to note that the type of methodologies that were utilized in measuring hormone concentrations, diabetes duration, dietary conditions (animal chow contains phytoestrogens), and cellular changes occurring due to chronic hyperglycemia versus estrogen treatment, may negatively influence the actual levels of hormones and/or their gastric receptors localized in enteric neurons. Finally, this may provide a misleading interpretation of the beneficial effects of these hormones on regulating gastric motility. In contrast, other studies have shown that, in general, gastric emptying is slower in young female rodents than age-matched male counterparts, and that estrogen may be the contributor for observed slower gastric emptying in both pregnant and non-pregnant rodents (reviewed in detail in [4]). Nevertheless, the above studies undoubtedly suggest that endogenous sex hormones may play an important role in gastric motility functions in females, and that a change in hormone levels and/or their receptor concentrations may drastically affect the stomach motility function that is seen in a diabetic or idiopathic setting [4].

However, as noticed with animal experiments, conflicting findings exist with regards to hormones and gut function in human studies. Estrogen and progesterone have inhibitory effects on smooth muscle of the lower esophageal sphincter, pylorus, and small bowel muscle strips, 
resulting in decreased gastrointestinal contractility $[4,8]$. Some studies suggest that gastric emptying is slower in females during the luteal phase of the menstrual cycle, when estrogen and progesterone levels are elevated, whereas others do not (reviewed in detail in [4]). Heitkemper et al. [9] demonstrated that the exacerbation of symptoms during the luteal phase in patients with irritable bowel syndrome. Recently, it has also been shown that a few gastroparesis symptoms were aggravated in younger women during the luteal phase of their menstrual cycle [10]. However, these studies did not observe gastroparesis symptoms in the women who are using contraceptives. Additional in-depth studies are required to discover whether endogenous sex hormones are beneficial or detrimental for stomach motility functions.

The role of nitric oxide has been well established in stomach motility functions [4]. nNOS is an inhibitory neurotransmitter and plays a critical role in stomach emptying. In a limited human study, the most common findings were the loss of ICC and the inhibition of nNOS expression in diabetic and/or idiopathic gastroparesis. Human studies are significantly limited by lack of access to prospectively collected tissue; therefore, much attention has been paid to rodent findings. Experiments conducted in animals revealed that nNOS dimerization is critical for gastric motility, and a change in nNOS function and expression may lead to delayed gastric emptying in diabetic, hyperlipidemia, oxidative stress, and, most importantly, chronic estrogen deficiency settings $[4,5]$. Several lines of evidence have revealed that estrogens elevate both eNOS and nNOS function, and maintain cardiovascular and neuronal functions, suggesting that nNOS function in stomach may be regulated by endogenous hormones [4, 11]. In support of this hypothesis, Shah et al. [12] demonstrated that estrogen supplementation improved nNOS expression, nNOS neurons, and, finally, nNOS function in adult female rats. The role and regulation of nNOS by gender, hormones, and, most importantly, by its cofactor tetrahydrobiopterin has been well documented and reviewed in the literature [4]. Oxidative stress is one of the detrimental factors in the pathogenesis of gastroparesis [13, 14]. Most importantly, estrogens are known to protect various cell types (including neuronal cells) against oxidative stress; however, the integral role of these hormones associated with oxidative stress-induced gastroparesis is yet to be demonstrated $[4,15]$. Finally, this editorial provide a caution that careful attention is essential when scientists work with hormones, because they could be either beneficial or detrimental depending upon the protocols utilized and doses tested in both animal and human studies.

Recent studies have also demonstrated that stomach parietal cells abundantly express aromatase, a key enzyme for the production of sex steroids, and catalyze the final step of the biosynthetic pathway of estrogen [16]. Though gastric aromatase/estrogen has been implicated in several pathophysiological states, such as liver regeneration and feminization, by acting as a local regulator of the gastrohepatic axis, its functional role has still to be elucidated. Premaratne et al. [17] reported that nNOS is expressed in rat parietal cells and may participate in the regulation of gastric acid secretions. It is possible that estrogen may influence gastric parietal cell secretion directly in association with nNOS, and/or indirectly by diffusing to and acting on adjacent inhibitory (n) NOS neurons and regulating gastric emptying. Future studies are warranted to investigate whether these signaling pathways are different from men versus women, and therefore responsible for gender differences observed in the gastric emptying process in both health and diabetes.

Funding US Department of Veterans Affairs.

\section{References}

1. Grover M, Farrugia G, Lurken MS, et al. Cellular changes in diabetic and idiopathic gastroparesis. Gastroenterology. 2011;140:1575-1585.

2. Oh JH, Pasricha PJ. Recent advances in the pathophysiology and treatment of gastroparesis. J Neurogastroenterol Motil. 2013;19: $18-24$.

3. Parkman HP, Yates K, Hasler WL, et al. Clinical features of idiopathic gastroparesis vary with sex, body mass, symptom onset, delay in gastric emptying, and gastroparesis severity. Gastroenterology. 2011;140:101-115.

4. Gangula PR, Sekhar K, Mukhopadhyay S. Gender bias in gastroparesis: is nitric oxide the answer? Dig Dis Sci. 2011;56: $2520-2527$.

5. Ravella K, Al-Hendy A, Sharan C, et al. Chronic estrogen deficiency causes gastroparesis by altering neuronal nitric oxide synthase function. Dig Dis Sci (Epub ahead of print). doi: 10.1007/s10620-013-2610-4.

6. Ali MS, Tiscareno-Grejada I, Locovei S, et al. Gender and estradiol as major factors in the expression and dimerization of $\mathrm{nNOS} \alpha$ in rats with experimental diabetic gastroparesis. Dig Dis Sci. 2012;57:2814-2825.

7. Mankhey RW, Bhatti F, Maric C. 17ß-Estradiol replacement improves renal function and pathology associated with diabetic nephropathy. Am J Physol Ren Physiol. 2005;288:F399-F405.

8. Fisher RS, Roberts GS, Grabowski CJ, Cohen S. Inhibition of LES circular muscle by female sex hormones. Am J Physiol. 1978;234:E243-E247.

9. Heitkemper MM, Cain KC, Jarrett ME, Burr RL, Hertig V, Bond EF. Symptoms across menstrual cycle in women with irritable bowel syndrome. Am J Gastroenterol. 2003;98:420-430.

10. Verrengia M, Sachdeva P, Gaughan J, Fisher RS, Parkman HP. Variation of symptoms during the menstrual cycle in female patients with gastroparesis. Neurogastroenterol Motil. 2011;23: 625.

11. Lekontseva O, Chakrabarti S, Jiang Y, Cheung CC, Davidge ST. Role of neuronal nitric oxide synthase in estrogen-induced relaxation in rat resistance arteries. J Pharmacol Exp Ther. 2011; 339:367-375.

12. Shah S, Nathan L, Singh R, Fu YS, Chaudhuri G. E2 and not P4 increases NO release from NANC nerves of the gastrointestinal 
tract: implications in pregnancy. Am J Physiol Regul Integr Comp Physiol. 2001;280:R1546-R1554.

13. Kashyap P, Farrugia G. Oxidative stress: key player of gastrointestinal complications of diabetes. Neurogastroenterol Motil. 2011;23:111-114.

14. Mukhopadhyay S, Sekhar KR, Hale AB, et al. Loss of NRF2 impairs gastric nitrergic stimulation and function. Free Radic Biol Med. 2011;51:619-625.

15. Numakawa T, Matsumoto T, Numakawa Y, Richards M, Yamawaki S, Kunugi H. Protective action of neurotrophic factors and estrogen against oxidative stress-mediated neurodegeneration. $J$ Toxicol. 2011;2011:405194.

16. Ozawa M, Takahashi K, Akazawa K, et al. PET of aromatase in gastric parietal cells using 11C-Vorozole. J Nucl Med. 2011; 52:1964-1969.

17. Premaratne S, Xue C, McCarty JM, et al. Neuronal nitric oxide synthase: expression in rat parietal cells. Am J Physiol Gastrointest Liver Physiol. 2001;280:G308-G313. 